\title{
Spinal Sagittal Alignment, Hospital Anxiety and Depression Scale Scores, and Patient-Reported Outcome among People with Sporting Activity
}

\author{
Shin $\mathrm{Oe}^{1}$, Yu Yamato ${ }^{1}$, Tomohiko Hasegawa ${ }^{2}$, Go Yoshida ${ }^{2}$, Sho Kobayashi ${ }^{3}$, Tatsuya Yasuda ${ }^{2}$, \\ Tomohiro Banno ${ }^{2}$, Hideyuki Arima ${ }^{2}$, Yuki Mihara ${ }^{2}$, Hiroki Ushirozako ${ }^{2}$, Tomohiro Yamada ${ }^{2}$, \\ Koichiro Ide ${ }^{2}$, Yuh Watanabe ${ }^{2}$, Haruo Niwa ${ }^{4}$, Yukihiro Matsuyama ${ }^{2}$ \\ ${ }^{I}$ Division of Geriatric Musculoskeletal Health, Department of Orthopedic Surgery, Hamamatsu University School of Medicine, Hamamatsu, Japan \\ ${ }^{2}$ Department of Orthopedic Surgery, Hamamatsu University School of Medicine, Hamamatsu, Japan \\ ${ }^{3}$ Department of Orthopedic Surgery, Hamamatsu Medical Center, Hamamatsu, Japan \\ ${ }^{4}$ Department of General Medicine, Toei Hospital, Toei, Japan
}

Study Design: Retrospective cohort study.

Purpose: This study aimed to investigate how participation in sporting activity affects patient-reported outcome (PRO), including Neck Disability Index (NDI), in males and females.

Overview of Literature: Previously, our study reported that factors with a negative influence on the NDI in females were a lack of sporting activities. However, it was still unclear why it affected poor scores of NDI.

Methods: The subjects were 473 volunteers. They were divided into two groups (activity and non-activity) according to participation or non-participation in sporting activities using a self-filled questionnaire. The evaluation items were height, weight, grip strength, bone density, Hospital Anxiety and Depression Scale (HADS) score, standing radiographic parameters, PRO (evaluated by EuroQol-5 dimension [EQ-5D], Oswestry Disability Index [ODI]), and NDI.

Results: There were 101 males in the non-activity group and 69 in the activity group and 178 females in the non-activity group and 125 in the activity group. For the males, the evaluation items with significant influence were cervical lordosis (non-activity group:activity group, $\left.17^{\circ}: 22^{\circ}\right)$ and $\mathrm{T} 1$ slope minus cervical lordosis $\left(10^{\circ}: 6^{\circ}, p<0.05\right)$. For the females, the evaluation items with significant influence were sagittal vertical axis (28:14 mm), HADS (10.4:8.4), EQ-5D (0.79:0.86), ODI (17:12), and NDI (12:9, $p<0.01)$. HADS and PRO in the females were significantly correlated with the EQ-5D (-0.40), ODI (0.43), and NDI (0.55).

Conclusions: Males who participated in sporting activities had better cervical spine alignment but no effect on PRO. Females with sporting activities had better spinal global alignment and less mental stress. It is suggested that sporting activity in females might be associated with PRO because HADS highly correlates with PRO.

Keywords: Sporting activity; Patients reported outcome; Spinal sagittal alignment; Hospital Anxiety and Depression Scale scores

Received Jun 28, 2019; Revised Aug 16, 2019; Accepted Sep 5, 2019

Corresponding author: Shin Oe

Division of Geriatric Musculoskeletal Health, Department of Orthopedic Surgery, Hamamatsu University School of Medicine, 1-20-1

Handayama Higashi-ku, Hamamatsu, Shizuoka 431-3192, Japan

Tel: +81-53-435-2299, Fax: +81-53-435-2296, E-mail: mecersior@gmail.com 


\section{Introduction}

Neck pain and low back pain are common complaints all over the world. Clinical practice guidelines recommend that people with neck pain or low back pain should undergo exercise therapy [1-4]. Oe et al. [5] reported the cut-off values and factors that negatively influenced the Neck Disability Index (NDI). This study suggested that the negative factors in males were manual work and cervical malalignment on a whole-spine standing radiograph and in females were lower body fat percentage, lower hand grip strength, and a lack of sporting activity. It is also reported that cervical sagittal malalignment on a wholespine standing radiograph deteriorates along with patientreported outcome (PRO) [6-8]. However, it remains unclear why females with no cervical malalignment show poor scores on the NDI.

It is well-known that neck pain is more frequent in females than in males [9-11]. Cagnie et al. [12] reported in their internet study that the incidence of neck pain in females (54.7\%; 123 of 225 office workers) was significantly higher than that in males (38.3\%; 110 of 287 office workers) and sex was a significant risk factor contributing to neck pain. Moreover, they reported that the incidence of neck pain among people who do not participate in sporting activities (56.7\%; 111 of 196 office workers) was also significantly higher than those who do participate in sporting activities (38.6\%; 122 of 316 office workers). However, there are very few studies that support the theory that sporting activity is effective for improving neck pain. Some studies though have demonstrated that psychosocial stress is also an important risk factor in neck pain and back pain $[9,10,13,14]$. The purpose of this study is to investigate how sporting activity can affect the parameters of whole-spine standing radiography, EuroQol-5 dimension (EQ-5D), Oswestry Disability Index (ODI), and NDI, as well as to assess psychosocial stress by sex.

\section{Materials and Methods}

\section{Institutional review board approval}

The study protocol was approved by the institutional review board of Hamamatsu University School of Medicine, Shizuoka, Japan (IRB approval no., 201201).

\section{Subjects}

The participants of this study were 870 volunteers who lived in Toei town in Aichi prefecture, Japan. They participated in a health screening study called the TOEI Study in 2016 after responding to public notices. They joined this study on a voluntary basis. All of the participants provided their written informed consent.

\section{Inclusion criteria}

The inclusion criteria were as follows: (1) age 40 years old or more and (2) informed consent for participation in this study.

\section{Exclusion criteria}

The exclusion criteria were as follows: (1) no available whole-spine radiographs in the standing position; (2) no questionnaire returns; (3) Cobb angle $>25^{\circ}$ in the coronal plane on radiographs; (4) symptomatic osteoporotic fractures with severe wedge-shaped vertebrae of grade 3 according to semi-quantitative grading [15]; and (5) history of instrumented spinal surgery or joint arthroplasty.

\section{Data collection and radiographic parameters}

The following demographic characteristics were evaluated: age, height $(\mathrm{cm})$, weight $(\mathrm{kg})$, body mass index (BMI, $\mathrm{kg} /$ $\mathrm{m}^{2}$ ), grip strength in right hand $(\mathrm{kg})$, hand grip strength, body composition (amount of muscle; $\mathrm{kg}$, percent of body fat, and basal metabolism; kcal/day), and bone mineral density (BMD). Body composition was measured using bioelectrical impedance analysis via a Tanita Dual-Body Composition Monitor (DC-430A; Tanita Corp., Tokyo, Japan). This device was validated for the precise evaluation of body composition [16]. BMD of the total proximal femur was measured using dual-energy X-ray absorptiometry and expressed as a percentage of the young adult mean (\%YAM). The total proximal femur was evaluated in this study because the lumbar spine was affected by degenerative changes, especially in the elderly. The questionnaire items were as follows: (1) sporting activity "Are you currently in the habit of participating in sports? What kind of sport is it?"; (2) number of births; (3) ODI, EQ-5D, and NDI for evaluating (PRO); and (4) Hospital Anxiety and Depression Scale (HADS) for evaluating mental status 

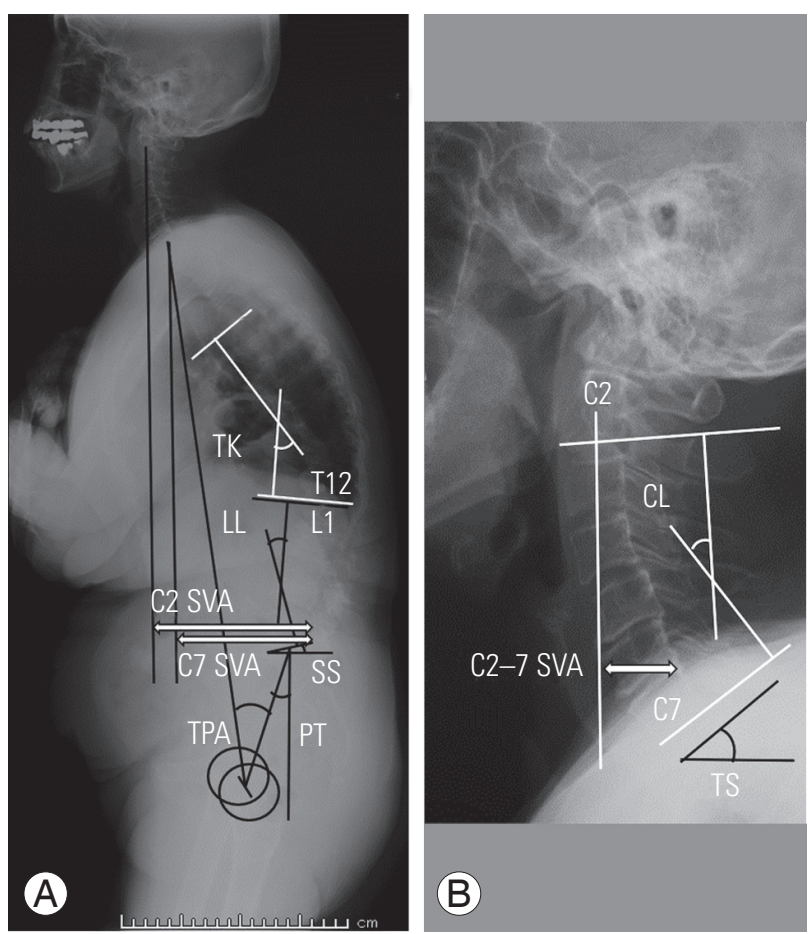

Fig. 1. (A, B) Evaluated radiographic parameters. SS, sacral slope; PT, pelvic tilt; LL, lumbar lordosis (L1-S1); TK, thoracic kyphosis (T5-T12); TS, T1 slope; CL, cervical lordosis (C2-C7); (7) SVA, sagittal vertical axis; TPA, T1 pelvic angle.

[17]. HADS is a 14-item scale in the form of a self-administered questionnaire. These items are divided into anxiety (HADS-A; seven items) and depression (HADS-D; seven items). Each item was scored on a 4-point scale from 0 (good) to 3 (bad). Possible total scores (HADS-T) range from 0 to 42 . Higher scores indicate a more severe anxiety or depression status. All volunteers answered these questionnaires when they attended the health screening.

The evaluated radiographic parameters were as follows (Fig. 1): (1) sacral slope (SS); (2) pelvic tilt (PT); (3) pelvic incidence (PI); (4) lumbar lordosis (LL; L1-S1); (5) PI minus LL (PI-LL); (6) thoracic kyphosis (TK; T5-T12); (7) T1 slope (TS); (8) cervical lordosis (CL; C2-C7); (9) TS minus CL (TS-CL); (10) C2-7 sagittal vertical axis (C2-7 SVA; distance between a plumb line from the center of the $\mathrm{C} 2$ vertebra and the posterior superior corner of C7); (11) C7 SVA; (12) C2 SVA; and (13) T1 pelvic angle (TPA; the angle between a line from the femoral heads to the center of the $\mathrm{T} 1$ vertebral body and a line from the femoral heads to the center of the superior sacral end plate). The digitized radiographs were transferred to an imaging software (Surgimap Spine; Nemaris Inc., New York, NY, USA), and each parameter was measured by seven board-certified

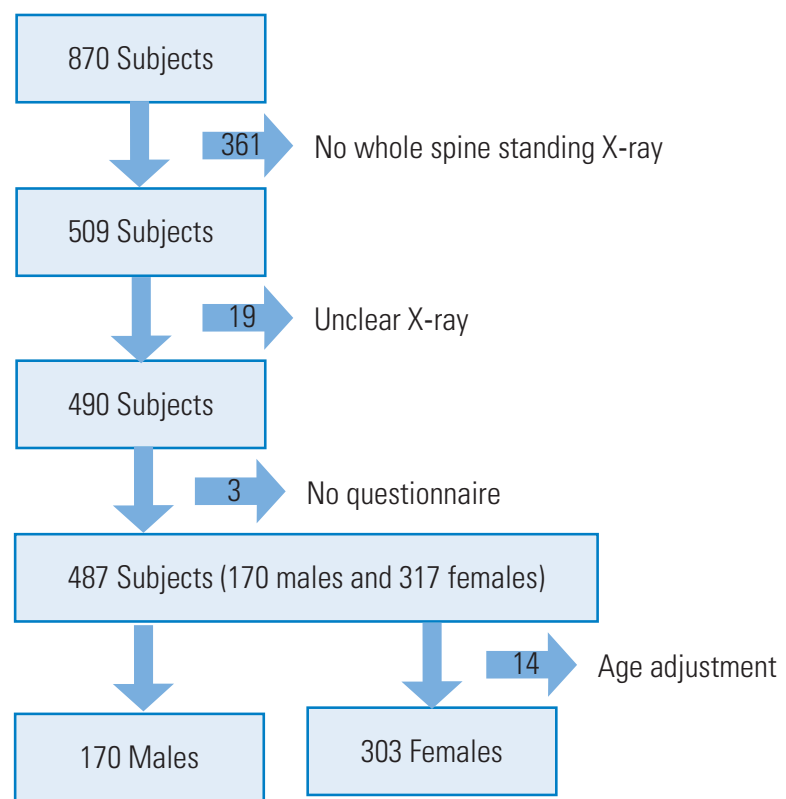

Fig. 2. Flowchart of volunteer recruitment. A total of 397 were excluded, and 170 males and 303 females were recruited.

spine surgeons in Hamamatsu University Hospital.

\section{Statistical analysis}

Statistical analyses were performed using IBM SPSS ver. 23.0 (IBM Corp., Armonk, NY, USA). The independent $t$-test or chi-square test was used to detect differences according to the sporting activity. The factors associated with a sporting activity were investigated using multiple logistic regression analysis. A $p$-value of $<0.05$ was considered statistically significant.

\section{Results}

The volunteer recruitment process is shown in Fig. 2. A total of 397 volunteers were excluded for the following reasons: no whole-spine standing radiograph $(\mathrm{n}=361$; they did not wish to undergo radiography); unclear radiograph $(\mathrm{n}=19$; it was impossible to evaluate the radiographic parameters); no questionnaire returns $(\mathrm{n}=3)$; and age adjustment (only 14 females). In the females, there was a gap of the age between the no sporting activity group (NS group, lower age) and the sporting activity group (S group, higher age). Therefore, 14 females were excluded for age matching in both groups. Finally, 170 males and 303 females were recruited for this study. 
Table 1. Demographic data among volunteers

\begin{tabular}{|c|c|c|c|c|c|c|}
\hline \multirow[b]{2}{*}{ Characteristic } & \multicolumn{3}{|c|}{ Male } & \multicolumn{3}{|c|}{ Female } \\
\hline & $\begin{array}{l}\text { Sports activity } \\
(-)\end{array}$ & $\begin{array}{l}\text { Sports activity } \\
\qquad(+)\end{array}$ & $p$-value & $\begin{array}{c}\text { Sports activity } \\
(-)\end{array}$ & $\begin{array}{l}\text { Sports activity } \\
(+)\end{array}$ & $p$-value \\
\hline No. of patients & 101 & 69 & & 178 & 125 & \\
\hline Age (yr) & $74.9 \pm 7.4$ & $74.6 \pm 8.0$ & 0.789 & $73.6 \pm 8.1$ & $73.9 \pm 8.5$ & 0.783 \\
\hline Height (cm) & $162.0 \pm 6.9$ & $161.6 \pm 7.9$ & 0.714 & $148.5 \pm 6.7$ & $149.7 \pm 5.9$ & 0.108 \\
\hline Weight $(\mathrm{kg})$ & $59.6 \pm 9.3$ & $61.3 \pm 7.6$ & 0.214 & $48.9 \pm 7.6$ & $50.6 \pm 7.4$ & 0.055 \\
\hline Body mass index $\left(\mathrm{kg} / \mathrm{m}^{2}\right)$ & $22.7 \pm 3.1$ & $23.5 \pm 2.3$ & 0.079 & $22.1 \pm 3.3$ & $22.6 \pm 3.1$ & 0.236 \\
\hline Grip strength (kg) & $35.7 \pm 7.6$ & $35.7 \pm 9.4$ & 0.966 & $23.6 \pm 4.7$ & $24.5 \pm 5.6$ & 0.125 \\
\hline Amount of muscle (kg) & $43.9 \pm 5.4$ & $44.6 \pm 4.8$ & 0.379 & $32.1 \pm 3.5$ & $32.6 \pm 3.6$ & 0.214 \\
\hline Percent of body fat (\%) & $20.3 \pm 5.4$ & $21.4 \pm 5.6$ & 0.184 & $28.3 \pm 7.0$ & $29.2 \pm 6.8$ & 0.247 \\
\hline Base metabolism (kcal/day) & $1,196.0 \pm 155.7$ & $1,221.2 \pm 171.4$ & 0.372 & $966.5 \pm 114.6$ & $983.7 \pm 112.3$ & 0.202 \\
\hline Young adult mean value ( $\%$, at total femur) & $78.9 \pm 11.9$ & $82.1 \pm 15.4$ & 0.155 & $70.9 \pm 14.2$ & $71.6 \pm 12.1$ & 0.642 \\
\hline No. of births & - & - & - & $2.4 \pm 0.9$ & $2.5 \pm 0.9$ & 0.349 \\
\hline HADS-anxiety & $4.2 \pm 3.1$ & $4.5 \pm 2.9$ & 0.523 & $6.1 \pm 3.2$ & $5.1 \pm 3.7$ & $0.024^{*}$ \\
\hline HADS-depression & $4.0 \pm 3.0$ & $3.7 \pm 3.2$ & 0.542 & $4.3 \pm 3.4$ & $3.3 \pm 3.3$ & $0.012^{*}$ \\
\hline HADS-total & $8.2 \pm 5.4$ & $8.2 \pm 5.4$ & 0.996 & $10.4 \pm 6.0$ & $8.4 \pm 6.4$ & $0.009^{* *}$ \\
\hline EuroQol-5 dimension & $0.828 \pm 0.174$ & $0.850 \pm 0.154$ & 0.400 & $0.787 \pm 0.164$ & $0.857 \pm 0.160$ & $0.000^{* * *}$ \\
\hline Oswestry Disability Index (\%) & $13.1 \pm 13.7$ & $11.8 \pm 12.9$ & 0.531 & $16.8 \pm 13.8$ & $11.8 \pm 12.0$ & $0.001^{* *}$ \\
\hline Neck Disability Index (\%) & $8.6 \pm 9.5$ & $9.2 \pm 8.6$ & 0.641 & $12.0 \pm 10.8$ & $8.9 \pm 8.7$ & $0.007^{* *}$ \\
\hline
\end{tabular}

Values are presented as number or mean \pm standard deviation.

HADS, Hospital Anxiety and Depression Scale.

${ }^{*} p<0.05$. ${ }^{* *} p<0.01 .{ }^{* * *} p<0.001$.

\section{Demographic data among volunteers}

Table 1 shows the demographic data among the volunteers by gender. There were 101 and 69 male volunteers in the NS group and S group, respectively. There were 178 and 125 female volunteers in the NS group and S group, respectively. In the NS group, the mean age of the males was 74.9 years and of the females 73.6 years. In the $S$ group, the mean age of the males was 74.6 years old and of the females 73.9 years, after age adjustment. There was no significant difference between the males in the $S$ group and the males in the NS group in terms of height, weight, BMI, grip strength, muscle mass, body fat percentage, basal metabolic rate, YAM value, and number of births. A similar result was found when the females in the $\mathrm{S}$ group were compared with the females in the NS group. Moreover, HADS-A, HADS-D, EQ-5D, ODI, and NDI did not differ in the males in the $S$ and NS groups. However, unlike the males, the females in the $S$ group had significantly better scores for HADS-A (NS group:S group,
6.1:5.1; $p=0.024$ ), HADS-D (NS group:S group, 4.3:3.3; $p=0.012$ ), HADS-T (NS group:S group, 10.4:8.4; $p=0.009$ ), EQ-5D (NS group:S group, 0.787:0.857; $p=0.000$ ), ODI (NS group:S group, $16.8 \%: 11.8 \% ; p=0.001$ ), and NDI (NS group:S group, $12.0 \%: 8.9 \% ; p=0.007)$.

\section{Radiographic parameters among volunteers}

Radiographic parameters in both males and females are shown in Table 2. There were no significant differences in lumbo-pelvic (SS, PT, PI, LL, and PI-LL), cervico-thoracic (TK and TS), and global alignment (C7 SVA, C2 SVA, and TPA) in the males in the $S$ and NS groups. However, there were significant differences in CL (NS group:S group, $17.4^{\circ}: 21.6^{\circ} ; p=0.044$ ) and TS-CL (NS group:S group, $9.8^{\circ}: 5.9^{\circ} ; p=0.040$ ), which represents cervical alignment.

In terms of the lumbo-pelvic (SS, PT, PI, LL, and PI-LL) and cervico-thoracic (TK, TS, CL, TS-CL, and C2-7 SVA) parameters, there were no significant differences in the females in the $S$ and NS groups. However, global align- 
Table 2. Radiographic parameters among volunteers

\begin{tabular}{|c|c|c|c|c|c|c|}
\hline \multirow{2}{*}{ Variable } & \multicolumn{3}{|c|}{ Male } & \multicolumn{3}{|c|}{ Female } \\
\hline & Sports activity (-) & Sports activity $(+)$ & $p$-value & Sports activity (-) & Sports activity $(+)$ & $p$-value \\
\hline No. of patients & 101 & 69 & & 178 & 125 & \\
\hline Sacral slope & $29.2 \pm 10.4$ & $31.3 \pm 9.2$ & 0.176 & $26.7 \pm 11.6$ & $28.5 \pm 10.5$ & 0.175 \\
\hline Pelvic tilt & $17.2 \pm 8.2$ & $17.2 \pm 9.4$ & 1.000 & $24.1 \pm 10.8$ & $23.2 \pm 9.3$ & 0.443 \\
\hline $\mathrm{Pl}$ & $46.4 \pm 9.9$ & $48.4 \pm 9.6$ & 0.172 & $50.8 \pm 11.1$ & $51.7 \pm 10.3$ & 0.493 \\
\hline $\mathrm{LL}$ & $40.7 \pm 14.7$ & $44.9 \pm 14.5$ & 0.068 & $40.1 \pm 20.5$ & $44.1 \pm 18.1$ & 0.078 \\
\hline $\mathrm{PI}-\mathrm{LL}$ & $5.7 \pm 13.4$ & $3.6 \pm 15.8$ & 0.356 & $10.8 \pm 19.9$ & $7.6 \pm 18.1$ & 0.158 \\
\hline Thoracic kyphosis & $30.3 \pm 10.8$ & $30.1 \pm 11.7$ & 0.906 & $32.9 \pm 14.5$ & $32.6 \pm 16.3$ & 0.869 \\
\hline TS & $27.2 \pm 8.7$ & $27.1 \pm 8.3$ & 0.910 & $26.6 \pm 11.7$ & $25.4 \pm 10.2$ & 0.370 \\
\hline $\mathrm{CL}$ & $17.4 \pm 12.2$ & $21.6 \pm 11.7$ & 0.044 & $25.4 \pm 15.5$ & $23.9 \pm 14.7$ & 0.389 \\
\hline TS-CL & $9.8 \pm 12.1$ & $5.9 \pm 12.3$ & 0.040 & $1.1 \pm 12.1$ & $1.5 \pm 12.7$ & 0.802 \\
\hline C2-7 SVA & $20.6 \pm 18.2$ & $19.0 \pm 15.4$ & 0.559 & $11.1 \pm 13.1$ & $10.2 \pm 13.9$ & 0.533 \\
\hline C7 SVA & $23.5 \pm 50.8$ & $18.5 \pm 54.7$ & 0.544 & $28.4 \pm 61.9$ & $14.0 \pm 49.5$ & $0.025^{*}$ \\
\hline C2 SVA & $41.5 \pm 57.4$ & $32.1 \pm 64.4$ & 0.315 & $36.6 \pm 67.8$ & $21.9 \pm 53.1$ & $0.036^{*}$ \\
\hline T1 pelvic angle & $16.1 \pm 10.1$ & $16.1 \pm 16.1$ & 0.971 & $21.0 \pm 13.7$ & $18.0 \pm 12.9$ & 0.053 \\
\hline
\end{tabular}

Values are presented as number or mean \pm standard deviation.

PI, pelvic incidence; LL, lumbar lordosis; TS, T1 slope; CL, cervical lordosis; SVA, sagittal vertical axis. * $p<0.05$.

ments, except for TPA, were significantly worse in the female NS group than in the female $\mathrm{S}$ group (C7 SVA, NS group:S group, 28.4:14.0 mm; $p=0.025$; C2 SVA, 36.6:21.9 $\mathrm{mm} ; p=0.036$ ).

\section{Significant factors correlated with sporting activity par- ticipation using multiple logistic regression analysis}

We used a multiple logistic regression analysis to identify the factors most affected by participation in sporting activities and to investigate why sporting activity was effective in females for a good PRO score. The dependent variables were set as participation in sporting activities and non-participation in sporting activities. In the males, the independent variables were set as LL, CL, and TS-CL based on the results shown in Tables 1 and 2. However, there were no significant factors associated with participation in sporting activities. In the females, the independent variables were set as HADS-A, HADS-D, HADS-T, C7 SVA, and C2 SVA. The only significant factor associated with participation in sporting activities in the females was HADS-T ( $p=0.010$; odds ratio, 0.950 ; $95 \%$ confidence interval, 0.913-0.987).

\section{Discussion}

Oe et al. [5] reported the factors associated with a negative influence on NDI, and our results suggested that in the males, these factors were cervical sagittal malalignment on the whole-spine standing radiograph, and in the females, lack of participation in sporting activities and the possibility of sarcopenia. There are many reports that sporting activities are effective for improving neck or back pain [1-4]. However, the purpose of this study was to clarify why a lack of participation in sporting activities was a factor in female NDI and not in males. As shown in Table 1, there was no significant difference in PRO (EQ$5 \mathrm{D}, \mathrm{ODI}, \mathrm{NDI})$ in males regardless of their participation or non-participation in sporting activities. On the other hand, females with sporting habits had significantly better outcomes on the EQ-5D, ODI, and NDI.

Details regarding the types of sporting activities by sex were shown in Figs. 3 and 4. Exercise intensity was evaluated using metabolic equivalent (MET) [18]. The mean exercise intensity was 4.0 METs in the males and 3.8 METs in the females. There was no significant difference with regard to the exercise intensity of each sporting activity undertaken by the males and females 


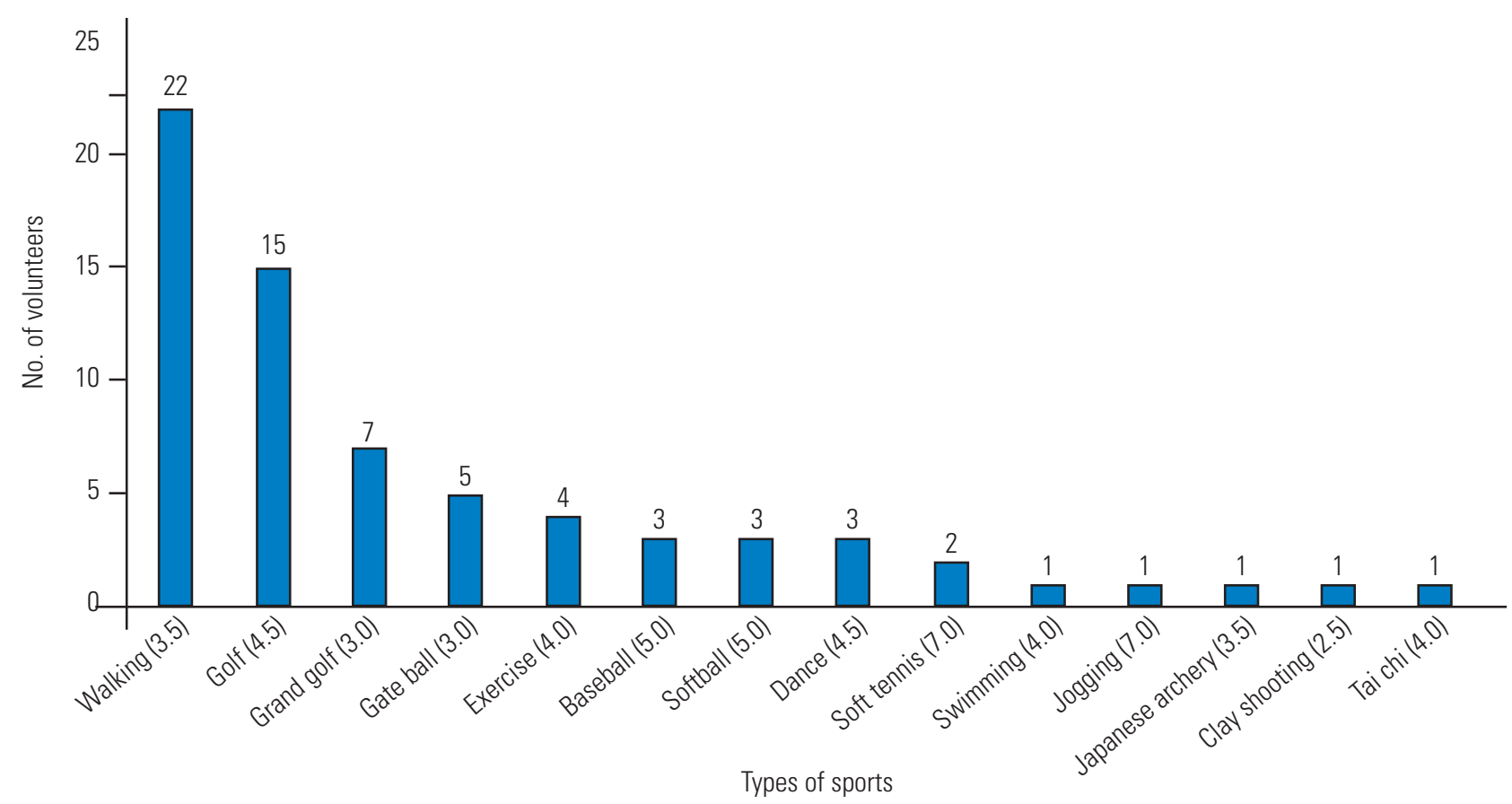

Fig. 3. Details of the types and exercise intensity of sports undertaken by males. Exercise intensity was evaluated using MET. Mean exercise intensity was 4.0 METs. MET, metabolic equivalent.

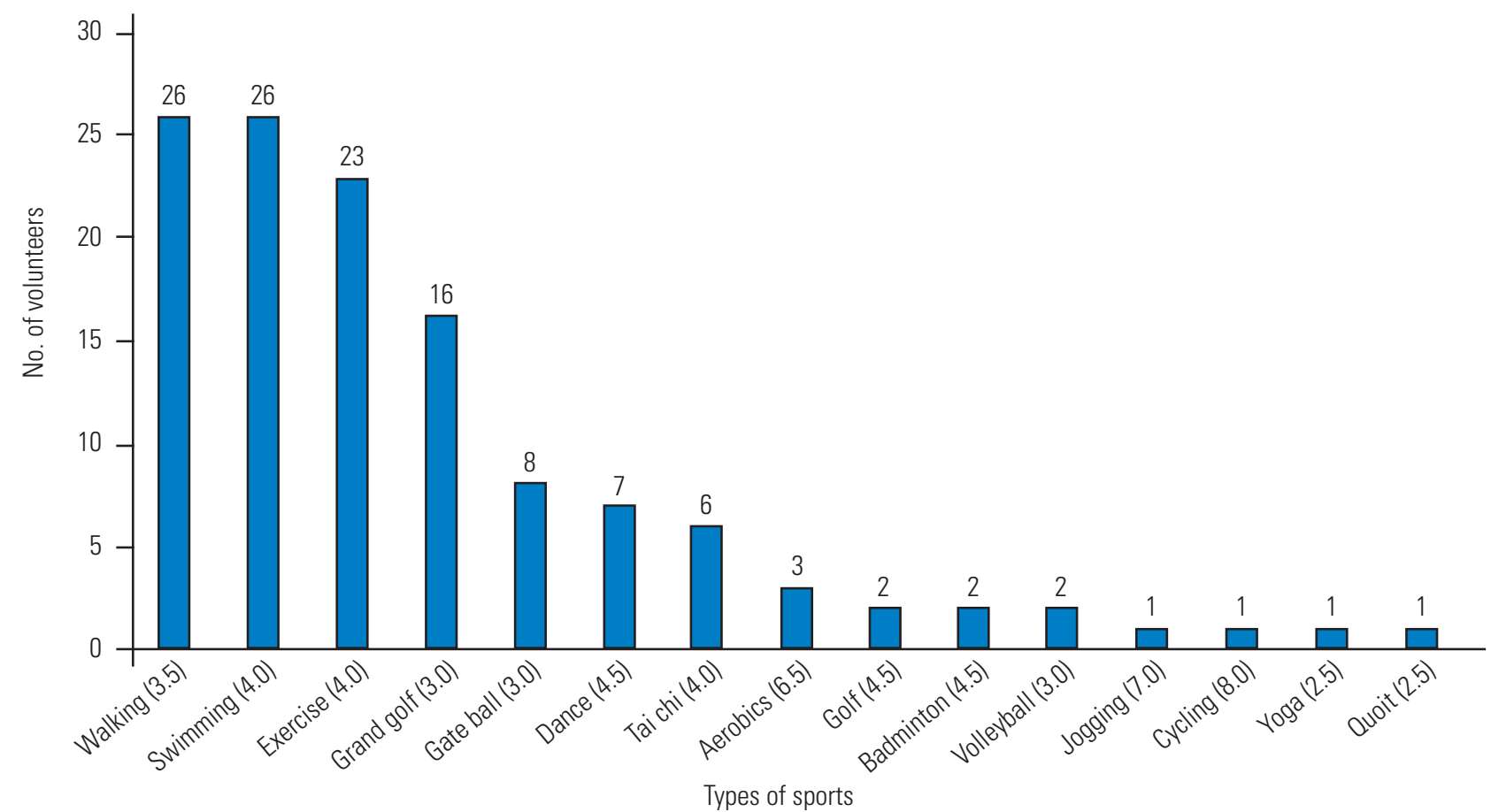

Fig. 4. Details of the types and exercise intensity of sports undertaken by females. Exercise intensity was evaluated using MET. Mean exercise intensity was 3.8 METs. MET, metabolic equivalent.

$(p=0.223)$. Despite this, we did find that HADS-T was the most relevant factor according to participation and nonparticipation in sporting activities in the females. Table 3 shows the correlation coefficients between HADS-T and PRO. In the males, the correlation coefficient with HADS$\mathrm{T}$ was -0.436 for EQ-5D, 0.441 for ODI, and 0.517 for 
Table 3. Coefficient of correlation between HADS-T and health-related quality of life

\begin{tabular}{lcc} 
Variable & HADS-T in males ( $p$-value) & HADS-T in females ( $p$-value) \\
Euro0ol-5 dimension & $-0.436(0.000)$ & $-0.396(0.000)$ \\
\hline Oswestry Disability Index & $0.441(0.000)$ & $0.431(0.000)$ \\
\hline Neck Disability Index & $0.517(0.000)$ & $0.546(0.000)$ \\
\hline
\end{tabular}

HADS-T, total score of Hospital Anxiety and Depression Scale.

NDI, whereas in the females, they were -0.396 for EQ-5D, 0.431 for ODI, and 0.546 for NDI. These results suggested that HADS-T was strongly correlated with all PRO. As shown in Table 1, the females who are engaged in sporting activities have significantly lower HADS-A, HADS-D, and HADS-T scores compared with the females who are not. However, no significant difference was observed for HADS-A, HADS-D, and HADS-T in the males, regardless of participation or non-participation in sporting activities. In the males, the mean HADS-T score was 8.2 in both the NS and S groups. In the females, the HADS-T scores were significantly higher in the NS group (10.4) than in the S group (8.4), which was similar to the HADS-T scores in the NS and $\mathrm{S}$ groups in males. In other words, as shown in Table 1, females initially have higher HADS-T scores than males, but participation in sporting activities may improve their mental statuses. As a result, this can make the PRO better. Indeed, it has been reported that sporting activities can improve anxiety and depression $[19,20]$. However, in the males, there was no change in the HADS score based on participation in sporting activities, but this result was because male HADS was not initially high. Therefore, we cannot conclude that participation in sporting activities is not effective for anxiety and depression in males.

In this study, we also investigated how participation in sporting activities influences various radiographic parameters (Table 2). In the males, TS-CL, which is an index of cervical malalignment, was significantly better in the $\mathrm{S}$ group. In the females, C2 SVA and C7 SVA, which are indexes of global alignment, were significantly better in the $S$ group. It might be effective to maintain muscle power due to sporting activity. Oe et al. [7] reported that deteriorated alignment originated from the cervical spine in males and from the pelvis in females. These results suggest that sporting activities may prevent deterioration of spinal malalignment with age.

This study has several limitations. First, 397 (45.6\%) of the 870 volunteers who participated were excluded.
The majority of these people (361 people) were excluded because they did not want to undergo whole-spine radiography. Radiography involves radiation exposure, which is why we could not strongly recommend radiographic examination. This exclusion might involve selection bias. Second, as the subjects were relatively elderly people living in mountainous areas, these results may not be generalizable to young people and those living in urban areas. Third, the data were based on the questionnaire of self-report. Therefore, the definition of sporting activity also depended on them. In other words, it was defined as sporting activity if volunteers considered it sporting activity. However, it was controversial whether low MET activity could be a sporting activity for training muscle because the actual frequency and intensity of the sporting activity were unclear. Fourth, spinal alignment was affected by not only sporting activity but also multiple factors (i.e., genetics, lifestyle, and work). Further study associated with spinal alignment should be necessary. Finally, in this study, we could not deny the possibility that people with good mental status liked to play sporting activities. Furthermore, longitudinal studies are required to investigate the changes that result from participation in sporting activities.

\section{Conclusions}

In conclusion, our results suggested that people with sporting activities had good spinal alignment, especially cervical spine alignment in males and global alignment in females. Sporting activities might also prevent deterioration of the spinal alignment with age. In addition, the HADS scores were significantly correlated with PRO. Therefore, females with sporting activities had good score of EQ-5D, ODI, and NDI because the HADS scores were significantly better. However, if PRO does not improve even after surgical treatment or medications, it is necessary to investigate the mental status of the patient. 


\section{Conflict of Interest}

No potential conflict of interest relevant to this article was reported. Shin Oe and Yu Yamato belong to a donated fund laboratory called Division of Geriatric Musculoskeletal Health.

\section{Acknowledgments}

Division of Geriatric Musculoskeletal Health received funding from Medtronic Sofamor Danek Inc., Japan Medical Dynamic Marketing Inc., and Meitoku Medical Institution Jyuzen Memorial Hospital. We have not received funding from the NIH, HHMI, or other such organizations.

\section{References}

1. Airaksinen O, Brox JI, Cedraschi C, et al. Chapter 4: European guidelines for the management of chronic nonspecific low back pain. Eur Spine J 2006;15 Suppl 2:S192-300.

2. Childs JD, Cleland JA, Elliott JM, et al. Neck pain: clinical practice guidelines linked to the International Classification of Functioning, Disability, and Health from the Orthopedic Section of the American Physical Therapy Association. J Orthop Sports Phys Ther 2008;38:A1-34.

3. Delitto A, George SZ, van Dillen L, et al. Low back pain. J Orthop Sports Phys Ther 2012;42:A1-57.

4. Qaseem A, Wilt TJ, McLean RM, Forciea MA; Clinical Guidelines Committee of the American College of Physicians. Noninvasive treatments for acute, subacute, and chronic low back pain: a clinical practice guideline from the American College of Physicians. Ann Intern Med 2017;166:514-30.

5. Oe S, Togawa D, Yoshida G, et al. Cut-off values of and factors associated with a negative influence on Neck Disability Index. Eur Spine J 2018;27:1423-31.

6. Tang JA, Scheer JK, Smith JS, et al. The impact of standing regional cervical sagittal alignment on outcomes in posterior cervical fusion surgery. Neurosurgery 2012;71:662-9.

7. Oe S, Togawa D, Nakai K, et al. The influence of age and sex on cervical spinal alignment among volunteers aged over 50. Spine (Phila Pa 1976) 2015;40:1487-94.
8. Oe S, Togawa D, Yoshida G, et al. Difference in spinal sagittal alignment and health-related quality of life between males and females with cervical deformity. Asian Spine J 2017;11:959-67.

9. Leclerc A, Niedhammer I, Landre MF, Ozguler A, Etore P, Pietri-Taleb F. One-year predictive factors for various aspects of neck disorders. Spine (Phila $\mathrm{Pa}$ 1976) 1999;24:1455-62.

10. Viikari-Juntura E, Martikainen R, Luukkonen R, Mutanen P, Takala EP, Riihimaki H. Longitudinal study on work related and individual risk factors affecting radiating neck pain. Occup Environ Med 2001;58:345-52.

11. Korhonen T, Ketola R, Toivonen R, Luukkonen R, Hakkanen M, Viikari-Juntura E. Work related and individual predictors for incident neck pain among office employees working with video display units. Occup Environ Med 2003;60:475-82.

12. Cagnie B, Danneels L, van Tiggelen D, de Loose V, Cambier D. Individual and work related risk factors for neck pain among office workers: a cross sectional study. Eur Spine J 2007;16:679-86.

13. Kato K, Sekiguchi M, Nikaido T, et al. Psychosocial stress after a disaster and low back pain-related interference with daily living among college students: a cohort study in Fukushima. Spine (Phila Pa 1976) 2017;42:1255-60.

14. Aasa U, Barnekow-Bergkvist M, Angquist KA, Brulin C. Relationships between work-related factors and disorders in the neck-shoulder and low-back region among female and male ambulance personnel. J Occup Health 2005;47:481-9.

15. Genant HK, Wu CY, van Kuijk C, Nevitt MC. Vertebral fracture assessment using a semiquantitative technique. J Bone Miner Res 1993;8:1137-48.

16. Ritchie JD, Miller CK, Smiciklas-Wright H. Tanita foot-to-foot bioelectrical impedance analysis system validated in older adults. J Am Diet Assoc 2005;105:1617-9.

17. Zigmond AS, Snaith RP. The hospital anxiety and depression scale. Acta Psychiatr Scand 1983;67:361-70.

18. Ainsworth BE, Haskell WL, Whitt MC, et al. Compendium of physical activities: an update of activity codes and MET intensities. Med Sci Sports Exerc 2000;32(9 Suppl):S498-504.

19. Mehnert A, Veers S, Howaldt D, Braumann KM, Koch U, Schulz KH. Effects of a physical exercise 
rehabilitation group program on anxiety, depression, body image, and health-related quality of life among breast cancer patients. Onkologie 2011;34:248-53.

20. Chen HM, Tsai CM, Wu YC, Lin KC, Lin CC. Ran- domised controlled trial on the effectiveness of home-based walking exercise on anxiety, depression and cancer-related symptoms in patients with lung cancer. Br J Cancer 2015;112:438-45. 